\title{
Nonfickian effect in time and space for diffusion processes
}

\author{
J. A. Ferreira G. Pena \\ CMUC, Department of Mathematics, University of Coimbra
}

\begin{abstract}
Diffusion processes are usually simulated using the classical diffusion equation. In certain scenarios such equation induces anomalous behaviour and consequently several improvements were introduced in the literature to overcome them. One of the most popular was the replacement of the diffusion equation by an integro-differential equation. Such equation can be established considering a modification of Fick's mass flux where a delay in time is introduced. In this paper, we consider mathematical models for diffusion processes that take into account a memory effect in time and space.
\end{abstract}

\section{Introduction}

The diffusion process is usually simulated using the classical diffusion equation

$$
\frac{\partial c}{\partial t}+\nabla \cdot(\mathbf{v} c)-\nabla \cdot\left(\mathbf{D}_{F} \nabla c\right)=f \text { in } \Omega \times(0, T],
$$

where $T$ is a positive real, $\Omega$ is a bounded domain in $\mathbb{R}^{n}, c$ denotes the concentration of a solute, $\mathbf{v}$ and $\mathbf{D}_{F}$ represent, respectively, the velocity field and the diffusion tensor and $f$ denotes a source term. Equation (1) is established using the mass conservation law

$$
\frac{\partial c}{\partial t}+\nabla \cdot \mathbf{J}_{t o t a l}=f
$$

where the total mass flux $\mathbf{J}_{\text {total }}$ is split into $\mathbf{J}_{\text {total }}=\mathbf{J}_{a}+\mathbf{J}_{F}$, with $\mathbf{J}_{a}$ being the advection flux

$$
\mathbf{J}_{a}=\mathbf{v} c
$$

and $\mathbf{J}_{F}$ given by Fick's law

$$
\mathbf{J}_{F}=-\mathbf{D}_{F} \nabla c .
$$

For instance, when diffusion processes occur in porous media, the diffusion tensor is replaced by

$$
\mathbf{D}_{F}=D_{m} \mathbf{I}+\mathbf{D}_{d}
$$

where $D_{m}$ is the molecular diffusion coefficient and $\mathbf{D}_{d}$ represents the dispersive tensor that depends on the velocity $\mathbf{v}$. We remark that the mass flux $\mathbf{J}_{F}$ can be split into $\mathbf{J}_{F}=\mathbf{J}_{m}+\mathbf{J}_{d}$ where

$$
\mathbf{J}_{m}=-D_{m} \nabla c
$$

is the mass flux defined by the molecular diffusion and $\mathbf{J}_{d}$ is the mass flux induced by the dispersion which in the Fick's context can be given by

$$
\mathbf{J}_{d}=-\mathbf{D}_{d} \nabla c .
$$


It was observed, in this case, that (1) provides accurate results in laboratory environments for perfectly homogeneous media and a deviation of the fickian behaviour occurs when nonhomogeneous media are used (see for instance $[8,12,11]$ ). Fick's law also does not reproduce flux behaviour in diffusion processes in biological tissues or polymeric materials. Indeed, it has been observed in this case that the flux at a certain time $t$ depends on the gradient of the concentration at a previous instant $t-\tau$ (see [2]). However, the main theoretical limitation induced by (1) is the infinite propagation speed which is not observable in real data sets and it is associated with its parabolic character.

To overcome the deviations observed when (1) is used, several approaches have been introduced in the literature. A common approach is to assume that the dispersive mass flux $\mathbf{J}_{d}(t)$ is related with the concentration $c$ at a delay time $t-\tau$, that is

$$
\mathbf{J}_{d}(t+\tau)=-\mathbf{D}_{d} \nabla c(t)
$$

where $\tau$ is a delay parameter. Then, Fick's law (6) can be approximated by

$$
\mathbf{J}_{d}+\tau \frac{\partial \mathbf{J}_{d}}{\partial t}=-\mathbf{D}_{d} \nabla c
$$

and from (2) we get

$$
\frac{\partial c}{\partial t}+\nabla \cdot(\mathbf{v} c)-\nabla \cdot\left(D_{m} \nabla c\right)-\frac{1}{\tau} \int_{0}^{t} e^{-\frac{t-s}{\tau}} \nabla \cdot\left(\mathbf{D}_{d} \nabla c(s)\right) d s=f \text { in } \Omega \times(0, T],
$$

provided that $\mathbf{J}_{d}(0)=0$. We remark that a three dimensional form of the nonfickian law of type (8) was proposed in [16]. Initial boundary value problems based on (9) were largely studied from a mathematical point of view. Without being exhaustive, we mention $[1,3,5,9,10,13,15,17$, $7,6]$.

An equation of the type of (9) was established in [18] using different arguments and with $\mathbf{J}_{d}=\beta_{1}\|\mathbf{v}\|^{2} \mathbf{I}+\beta_{2} \mathbf{v}^{t} \mathbf{v}$. This remark allows us to establish that the delay approach for the dispersion mass flux, at least in some convenient context, leads to the proposal introduced in [18].

Under convenient smoothness assumptions, equation (9) is equivalent to the following hyperbolic equation

$$
\tau \frac{\partial^{2} c}{\partial t^{2}}+\frac{\partial c}{\partial t}+\nabla \cdot\left(\tau \mathbf{v} \frac{\partial c}{\partial t}+\mathbf{v} c\right)-\nabla \cdot\left(\mathbf{D}_{F} \nabla c\right)-\nabla \cdot\left(D_{m} \nabla \frac{\partial c}{\partial t}\right)=\tau \frac{\partial f}{\partial t}+f \text { in } \Omega \times(0, T] .
$$

It is clear that if $\tau=0$ then (10) reduces to (1).

To simplify the presentation in what follows, $\mathbf{J}_{d}$ is represented only by $\mathbf{J}$ and $\mathbf{D}_{d}$ shall be denoted as $\mathbf{D}_{n F}$, the nonfickian diffusion tensor. As introduced in [8], let us suppose that $\mathbf{J}$ at a point $\mathbf{x}+\tau \mathbf{V} \mathbf{J}$ at time $t+\tau$ depends on the behaviour of the gradient of the concentration in $\mathbf{x}$ at time $t$, more precisely, let us assume that

$$
\mathbf{J}\left(\mathbf{x}+\tau \mathbf{v}_{\mathbf{J}}, t+\tau\right)=-\mathbf{D}_{n F} \nabla c(\mathbf{x}, t),
$$

which can be approximated by

$$
\tau \frac{\partial \mathbf{J}}{\partial t}+\tau\left(\mathbf{v}_{\mathbf{J}} \cdot \nabla\right) \mathbf{J}+\mathbf{J}=-\mathbf{D}_{n F} \nabla c .
$$

In (12), $\mathbf{D}_{n F}$ represents the dispersion tensor which is responsible by the nonfickian behaviour and $\mathbf{v}_{\mathbf{J}}$, that represents a nonfickian flux velocity, is a vector function depending only on $\mathbf{x}$. 
To model the evolution of a concentration in a one-dimensional porous medium, an hyperbolic equation can be obtained from (12) and the initial mass conservation law,

$$
\frac{\partial c}{\partial t}+\nabla \cdot(\mathbf{v} c)-\nabla \cdot\left(\mathbf{D}_{F} \nabla c\right)+\nabla \cdot \mathbf{J}=f \text { in } \Omega \times(0, T]
$$

which was proposed in [14]. We point out that such hyperbolic equation was not obtained using explicitly (12) nor the last expression was not mentioned in [14]. We point the reader to [8] for an overview on these models.

Following similar techniques that were used to deduce equations (9) and (10), under suitable regularity conditions, different (integro-)differential equations can be obtained from (12)-(13). These formulations are satisfied by the same solution as (12)-(13) but given the nature of the equations, new energy estimates can be proved and different quantities can be bounded.

A first possibility that we can follow to obtain a mathematical problem equivalent to the system (12), (13) is to solve the mass flux equation (12) using the method of characteristics. In fact, (12) can be rewritten in the following equivalent form

$$
\left\{\begin{array}{l}
t^{\prime}=\tau, \\
\mathbf{x}^{\prime}=\tau \mathbf{v} \mathbf{J}, \\
\mathbf{J}^{\prime}+\mathbf{J}=-\mathbf{D}_{n F} \nabla c .
\end{array}\right.
$$

The existence of the dispersive mass flux is guaranteed, at least locally, provided that $\mathbf{v} \mathbf{J}$ and $\mathbf{D}_{n F} \nabla c$ are Lipschitz functions. In this case, after solving the initial value problem associated with (14), the expression of $\mathbf{J}$ is obtained in function of $\mathbf{D}_{n F} \nabla c$. Let us consider now the simplest case: $\mathbf{v}_{\mathbf{J}}=$ Const. In this case using the method of characteristics in (12), we obtain for the dispersion mass flux the following representation

$$
\mathbf{J}(\mathbf{x}, t)=e^{-\frac{t}{\tau}} \mathbf{J}(\mathbf{x}, 0)-\frac{1}{\tau} \int_{0}^{t} e^{-\frac{t-s}{\tau}} \mathbf{D}_{n F} \nabla c\left(\mathbf{x}+\mathbf{v}_{\mathbf{J}}(s-t), s\right) d s .
$$

In this case, with $\Omega^{*}=\left\{\mathbf{x} \in \Omega: \mathbf{x}-t \mathbf{v}_{\mathbf{J}} \in \Omega\right\}$, and considering the mass conservation law (13), equation (9) is replaced by

$$
\begin{gathered}
\frac{\partial c}{\partial t}+\nabla \cdot(\mathbf{v} c)-\nabla \cdot\left(\mathbf{D}_{F} \nabla c\right)+\nabla \cdot \mathbf{J}(0) e^{-\frac{t}{\tau}} \\
-\frac{1}{\tau} \int_{0}^{t} e^{-\frac{t-s}{\tau}} \nabla \cdot\left(\mathbf{D}_{n F} \nabla c(\mathbf{x}+\mathbf{v} \mathbf{J}(s-t), s)\right) d s=f \text { in } \Omega^{*} \times(0, T],
\end{gathered}
$$

which requires smoothness on $\mathbf{J}$ at $t=0$.

It is clear that (14) admits an explicit expression for the dispersion mass flux $\mathbf{J}$ in simple cases. Moreover, in such cases, an integral representation for the dispersive mass flux of the type of (15) can be obtained. However, such representation puts a set of significant computational difficulties beyond the smoothness requirement for the $\mathbf{J}$ at initial time, like the computational cost of the integral term, the use of spatial grid that depends on time and that needs to be defined in each time level.

Another possibility is the replacement of (12), (13) by an hyperbolic equation as the one proposed in [14]. From (13) we obtain

$$
\tau \frac{\partial^{2} c}{\partial t^{2}}+\tau \nabla \cdot\left(\mathbf{v} \frac{\partial c}{\partial t}\right)-\tau \nabla \cdot\left(\mathbf{D}_{F} \nabla \frac{\partial c}{\partial t}\right)+\tau \nabla \cdot \frac{\partial \mathbf{J}}{\partial t}=\tau \frac{\partial f}{\partial t}
$$


From (12) we get

$$
\tau \nabla \cdot \frac{\partial \mathbf{J}}{\partial t}=-\tau \nabla \cdot\left(\left(\mathbf{v}_{\mathbf{J}} \cdot \nabla\right) \mathbf{J}\right)-\nabla \cdot \mathbf{J}-\nabla \cdot\left(\mathbf{D}_{n F} \nabla c\right)
$$

where

$$
\nabla \cdot \mathbf{J}=-\frac{\partial c}{\partial t}-\nabla \cdot(\mathbf{v} c)+\nabla \cdot\left(\mathbf{D}_{F} \nabla c\right)+f
$$

being this last representation deduced from (13). Furthermore, equation (13) enable us write

$$
\tau \nabla \cdot\left(\mathbf{v}_{\mathbf{J}} \nabla \cdot \mathbf{J}\right)=-\tau \nabla \cdot\left(\mathbf{v}_{\mathbf{J}} \frac{\partial c}{\partial t}\right)-\tau \nabla \cdot\left(\mathbf{v}_{\mathbf{J}} \nabla \cdot(\mathbf{v} c)\right)-\tau \nabla \cdot\left(\mathbf{v}_{\mathbf{J}} \nabla \cdot\left(\mathbf{D}_{F} \nabla c\right)\right)+\tau \nabla \cdot\left(\mathbf{v}_{\mathbf{J}} f\right) .
$$

If

$$
\nabla \cdot\left(\mathbf{v}_{\mathbf{J}} \nabla \cdot \mathbf{J}\right)=\nabla \cdot\left(\left(\mathbf{v}_{\mathbf{J}} \cdot \nabla\right) \mathbf{J}\right)
$$

then from (17)-(20) we conclude

$$
\begin{aligned}
& \tau \frac{\partial^{2} c}{\partial t^{2}}+\tau \nabla \cdot\left(\left(\mathbf{v}_{\mathbf{J}}+\mathbf{v}\right) \frac{\partial c}{\partial t}\right)-\tau \nabla \cdot\left(\mathbf{D}_{F} \nabla \frac{\partial c}{\partial t}\right)-\tau \nabla \cdot\left(\mathbf{v}_{\mathbf{J}} \nabla \cdot\left(\mathbf{D}_{F} \nabla c\right)\right. \\
& +\tau \nabla \cdot\left(\mathbf{v}_{\mathbf{J}} \nabla \cdot(\mathbf{v} c)\right)-\nabla \cdot\left(\left(\mathbf{D}_{F}+\mathbf{D}_{n F}\right) \nabla c\right) \\
& +\frac{\partial c}{\partial t}+\nabla \cdot(\mathbf{v} c)=f+\tau \frac{\partial f}{\partial t}+\tau \nabla \cdot\left(\mathbf{v}_{\mathbf{J}} f\right) \text { in } \Omega \times(0, T] .
\end{aligned}
$$

We point out that the establishment of (22) requires the condition (21), smooth data and it should be complemented with the initial conditions

$$
\left\{\begin{array}{l}
\frac{\partial c}{\partial t}(0)=-\nabla \cdot \mathbf{J}_{0}+f(0)+\nabla \cdot\left(\mathbf{D}_{F} \nabla c_{0}\right) \\
c(0)=c_{0} .
\end{array}\right.
$$

We remark that for pure nonfickian diffusion processes, equation (22) takes the form

$$
\begin{aligned}
\tau \frac{\partial^{2} c}{\partial t^{2}}+\tau \nabla \cdot( & \left.\left(\mathbf{v}_{\mathbf{J}}+\mathbf{v}\right) \frac{\partial c}{\partial t}\right)+\tau \nabla \cdot\left(\mathbf{v}_{\mathbf{J}} \nabla \cdot(\mathbf{v} c)\right) \\
& \left.-\nabla \cdot\left(\mathbf{D}_{n F}\right) \nabla c\right)+\frac{\partial c}{\partial t}+\nabla \cdot(\mathbf{v} c)=f+\tau \frac{\partial f}{\partial t}+\tau \nabla \cdot\left(\mathbf{v}_{\mathbf{J}} f\right) \quad \text { in } \Omega \times(0, T] .
\end{aligned}
$$

In conclusion, to model the diffusion processes that occurs in a bounded domain $\Omega \subset \mathbb{R}^{n}$, with fickian behaviour and nonfickian behaviour characterized by memory in time and in space, we consider the differential system (12), (13) which is complemented with initial data

$$
c(\mathbf{x}, 0)=c_{0}(\mathbf{x}) \text { and } \mathbf{J}(\mathbf{x}, 0)=\mathbf{J}_{0}(\mathbf{x}), \mathbf{x} \in \Omega .
$$

Regarding boundary conditions, let us first introduce a disjoint decomposition of the boundary: $\partial \Omega=\Gamma_{D} \cup \Gamma_{N}$ and denote $\mathbf{n}(\mathbf{x})$ (or simply $\mathbf{n}$ ) the unit outer normal vector at $\mathbf{x} \in \partial \Omega$. We assume that $\Gamma_{N}$ is isolated, this means that we do not have any mass flux, and in $\Gamma_{D}$ we know the concentration $c$

$$
\mathbf{J}_{\text {total }} \cdot \mathbf{n}=0 \text { on } \Gamma_{N}, \quad c=c_{n} \text { on } \Gamma_{D}
$$


where $\mathbf{J}_{\text {total }}$ represents the total mass flux

$$
\mathbf{J}_{\text {total }}=-\mathbf{D}_{F} \nabla c+\mathbf{v} c+\mathbf{J} .
$$

In what follows we assume that $c_{0}=0$, otherwise a change of variables needs to be considered which turns our model more complex. To summarize we remark that the coupled system (12),(13) comprises three components for the mass flux: the advective mass flux, the fickian mass flux (modeling, for instance, the molecular mass flux) and a nonfickian component (through (12)).

In this paper our aim is to study, from an analytical and numerical view point, the coupled model (12), (13) with convenient boundary and initial conditions. The paper is organized as follows: in Section 2, we obtain energy estimates for the proposed model as well as for the equivalent formulations introduced in Section 1; finally, in Section 3, we simulate the evolution of the coupled model and illustrate the different behaviour of the variables.

\section{Energy estimates}

In this section we focus on obtaining an energy estimate initial boundary value nonfickian problem $(12),(13),(25),(26)$. We also establish an energy estimate for an equivalent pure nonfickian hyperbolic problem to clarify the properties of the solution of the coupled problem (12)-(13).

\subsubsection{Some notations}

Let us first introduce some notations, necessary for the following sections. We denote by $L^{2}(\Omega)$, $H^{1}(\Omega)$ and more generally $W^{k, p}(\Omega)$ the standard Sobolev spaces of scalar functions defined in $\Omega$ and $L^{2}(\partial \Omega)$ denotes the usual $L^{2}$ space of functions defined on $\partial \Omega$. Given a nonzero measure portion $\Gamma$ of $\partial \Omega, H_{\Gamma}^{1}(\Omega)$ denotes the space of $H^{1}(\Omega)$ functions that have zero trace on $\Gamma$. Also, the equivalent spaces for vectorial functions are represented using the same notation, but with bold letters. With an abuse of notation, we shall denote by the same notation, $(\cdot, \cdot)$, the inner product of $L^{2}$ and $\mathbf{L}^{2}$.

Given a space $V$ as any of the ones introduces before, we define

$$
L^{2}(0, T ; V)=\left\{u:(0, T) \longrightarrow V: \int_{0}^{T}\|u(s)\|_{V}^{2} d s<\infty\right\}
$$

and

$$
H^{1}(0, T ; V)=\left\{u \in L^{2}(0, T ; V): \frac{\partial u}{\partial t} \in L^{2}(0, T ; V)\right\} .
$$

The two previous spaces are Banach spaces with the following norms:

$$
\|u\|_{L^{2}(0, T ; V)}^{2}=\int_{0}^{T}\|u(s)\|_{V}^{2} d s, \quad\|u\|_{H^{1}(0, T ; V)}^{2}=\int_{0}^{T}\left(\|u(s)\|_{V}^{2}+\left\|u^{\prime}(s)\right\|_{V}^{2}\right) d s .
$$

For vectorial functions, the definitions follow the same notation as for the scalar counterparts. The space $C([0, T] ; V)$ shall denote the subspace of $L^{2}(0, T ; V)$ of continuous functions in time.

Finally, given $\mathbf{v}_{\mathbf{J}} \in \mathbf{W}^{1, \infty}(\Omega)$ and $u \in H^{1}(\Omega)$ (or $\mathbf{u}^{2} \in \mathbf{H}^{1}(\Omega)$ ), we use the notation

$$
|u|_{\mathbf{v}_{\mathbf{J}}, \partial \Omega}^{2}=\int_{\partial \Omega} u^{2} \mathbf{v}_{\mathbf{J}} \cdot \mathbf{n} d s \quad\left(\text { or }|\mathbf{u}|_{\mathbf{v}_{\mathbf{J}}, \partial \Omega}^{2}=\int_{\partial \Omega}|\mathbf{u}|^{2} \mathbf{v}_{\mathbf{J}} \cdot \mathbf{n} d s\right) .
$$


Of course that if

$$
\mathbf{v}_{\mathbf{J}} \cdot \mathbf{n} \geq 0 \text { a.e. on } \partial \Omega,
$$

then $|\cdot|_{\mathbf{v}_{\mathbf{J}}, \partial \Omega}^{2}$ defines a seminorm in $L^{2}(\partial \Omega)$.

In what follows we assume that the matrices $\mathbf{D}_{F}$ and $\mathbf{D}_{n F}$ that arise in (12), (13) are real symmetric matrices a.e. in $\Omega$ and there exist uniform bounds $\alpha_{F}, \beta_{n F}, \alpha_{n F}, \beta_{n F}>0$ such that

$$
\alpha_{k}|\boldsymbol{\xi}|^{2} \leqslant \boldsymbol{\xi}^{T} \mathbf{D}_{k}(\mathbf{x}) \boldsymbol{\xi} \leqslant \beta_{k}|\boldsymbol{\xi}|^{2}, \forall \boldsymbol{\xi} \in \mathbb{R}^{d}, \forall \mathbf{x} \text { in } \Omega
$$

with $k \in\{n, n F\}$, where $|\cdot|$ denotes the Euclidean norm. In the following sections, to obtain energy estimates, we shall assume that (27) holds.

\subsection{Coupled model}

For the coupled problem, we assume that $\mathbf{v}_{\mathbf{J}} \in \mathbf{W}^{1, \infty}(\Omega)$ and $\mathbf{v}=\mathbf{0}$ (pure diffusion problem). The mixed weak formulation for the coupled model reads as: given $f \in L^{2}\left(0, T ; L^{2}(\Omega)\right), c_{0} \in$ $L^{2}(\Omega), \mathbf{J}_{0} \in \mathbf{L}^{2}(\Omega)$ find $c \in L^{2}\left(0, T ; H_{\Gamma_{D}}^{1}(\Omega)\right), \mathbf{J} \in L^{2}\left(0, T ; \mathbf{H}^{1}(\Omega)\right)$ such that $\frac{\partial c}{\partial t} \in L^{2}\left(0, T ; L^{2}(\Omega)\right)$, $\frac{\partial \mathbf{J}}{\partial t} \in L^{2}\left(0, T ; \mathbf{L}^{2}(\Omega)\right)$ and

$$
\begin{aligned}
\left(\frac{\partial c}{\partial t}(t), v\right)+\left(\mathbf{D}_{F} \nabla c(t), \nabla v\right)-(\mathbf{J}(t), \nabla v) & =(f(t), v) & \text { a.e. in }(0, T), \quad \forall v \in H_{\Gamma_{D}}^{1}(\Omega) \\
\tau\left(\frac{\partial \mathbf{J}}{\partial t}(t), \mathbf{w}\right)+(\mathbf{J}(t), \mathbf{w}) & & \\
+\tau\left(\left(\mathbf{v}_{\mathbf{J}} \cdot \nabla\right) \mathbf{J}(t), \mathbf{w}\right) & =-\left(\mathbf{D}_{n F} \nabla c(t), \mathbf{w}\right) & \text { a.e. in }(0, T), \quad \forall \mathbf{w} \in \mathbf{H}^{1}(\Omega) .
\end{aligned}
$$

Before the establishment of an energy estimate for this problem, let us denote by $\alpha_{n F,-1}$ and $\beta_{n F,-1}$ the constants in (28) that make the inequalities hold for $\mathbf{D}_{n F}^{-1}$. Let $d_{i j}$ denote the matrix entries of $\mathbf{D}_{n F}^{-1}$.

Proposition 1. Let $f \in L^{2}\left(0, T ; L^{2}(\Omega)\right), c_{0} \in L^{2}(\Omega)$ and $\mathbf{J}_{0} \in \mathbf{L}^{2}(\Omega)$. If $d_{i j} \in W^{1, \infty}(\Omega)$ and the matrix

$$
\mathbf{B}\left(\mathbf{v}_{\mathbf{J}}, \mathbf{D}_{n F}^{-1}\right):=\left[\operatorname{div}\left(d_{i j} \mathbf{v}_{\mathbf{J}}\right)\right]_{i j}
$$

is negative semidefinite a.e. in $\Omega$, then the solution $(c, \mathbf{J})$ of $(29)$ in $C\left([0, T] ; L^{2}(\Omega)\right) \times C\left([0, T] ; \mathbf{L}^{2}(\Omega)\right)$ verifies the following estimate:

$$
\begin{gathered}
\|c(t)\|_{0}^{2}+\tau \alpha_{n F,-1}\|\mathbf{J}(t)\|_{0}^{2}+\alpha_{F} \int_{0}^{t}\|\nabla c(s)\|_{0}^{2} d s+2 \alpha_{n F,-1} \int_{0}^{t}\|\mathbf{J}(s)\|_{0}^{2} d s \\
\leqslant\left\|c_{0}\right\|_{0}^{2}+\tau \alpha_{n F,-1}\left\|\mathbf{J}_{0}\right\|_{0}^{2}+\epsilon \int_{0}^{t}\|f(s)\|_{0}^{2} d s
\end{gathered}
$$

for some positive constant $\epsilon$.

Proof. We start by taking $v=c(t)$ in the first equation of (29). Using (28) it follows that

$$
\frac{1}{2} \frac{d}{d t}\|c(t)\|_{0}^{2}+\alpha_{F}\|\nabla c(t)\|_{0}^{2} \leqslant(\mathbf{J}(t), \nabla c(t))+(f(t), c(t)) .
$$

Applying Cauchy-Schwarz's and Young's inequality, we deduce, for all $\epsilon>0$,

$$
\frac{1}{2} \frac{d}{d t}\|c(t)\|_{0}^{2}+\alpha_{F}\|\nabla c(t)\|_{0}^{2} \leqslant \frac{\epsilon}{2}\|f(t)\|_{0}^{2}+\frac{1}{2 \epsilon}\|c(t)\|_{0}^{2}+(\nabla c(t), \mathbf{J}(t)) .
$$


On the other hand, taking $\mathbf{w}=\mathbf{D}_{n F}^{-1} \mathbf{J}(t)$ in the second equation of (29), we immediately conclude that

$$
\tau\left(\mathbf{D}_{n F}^{-1} \frac{\partial \mathbf{J}}{\partial t}(t), \mathbf{J}(t)\right)+\left(\mathbf{D}_{n F}^{-1} \mathbf{J}(t), \mathbf{J}(t)\right)+\tau\left(\mathbf{D}_{n F}^{-1}(\mathbf{v} \mathbf{J} \cdot \nabla) \mathbf{J}(t), \mathbf{J}(t)\right)=-(\nabla c(t), \mathbf{J}(t)) .
$$

It follows from (28) for the inverses that

$$
\alpha_{n F,-1}\|\mathbf{J}(t)\|_{0}^{2} \leqslant\left(\mathbf{D}_{n F}^{-1} \mathbf{J}(t), \mathbf{J}(t)\right) .
$$

For $t \in(0, T)$, let

$$
I_{1}(t)=\left(\mathbf{D}_{n F}^{-1} \frac{\partial \mathbf{J}}{\partial t}(t), \mathbf{J}(t)\right) \text { and } I_{2}(t)=\left(\mathbf{D}_{n F}^{-1}\left(\mathbf{v}_{\mathbf{J}} \cdot \nabla\right) \mathbf{J}(t), \mathbf{J}(t)\right) .
$$

Since $\mathbf{D}_{n F}^{-1}$ does not depend on $t$, it is straightforward to conclude that

$$
I_{1}(t)=\left(\mathbf{D}_{n F}^{-1} \frac{\partial \mathbf{J}}{\partial t}(t), \mathbf{J}(t)\right)=\frac{1}{2} \frac{d}{d t}\left(\mathbf{D}_{n F}^{-1} \mathbf{J}(t), \mathbf{J}(t)\right)
$$

On the other hand, using integration by parts we conclude that

$$
I_{2}(t)=\left(\mathbf{D}_{n F}^{-1}\left(\mathbf{v}_{\mathbf{J}} \cdot \nabla\right) \mathbf{J}(t), \mathbf{J}(t)\right)=\frac{1}{2} \int_{\partial \Omega}\left(\mathbf{D}_{n F}^{-1} \mathbf{J}(t)\right) \cdot \mathbf{J}(t)\left(\mathbf{n} \cdot \mathbf{v}_{\mathbf{J}}\right) d s-\frac{1}{2}\left(\mathbf{B}\left(\mathbf{v}_{\mathbf{J}}, \mathbf{D}_{n F}^{-1}\right) \mathbf{J}(t), \mathbf{J}(t)\right) .
$$

Combining (34), (35) and (36) in (33)

$$
\frac{\tau}{2} \frac{d}{d t}\left(\mathbf{D}_{n F}^{-1} \mathbf{J}(t), \mathbf{J}(t)\right)+\alpha_{n F,-1}\left(\|\mathbf{J}(t)\|_{0}^{2}+\frac{\tau}{2}|\mathbf{J}(t)|_{\mathbf{v}_{\mathbf{J}}, \partial \Omega}^{2}\right) \leqslant-(\nabla c(t), \mathbf{J}(t))+\frac{\tau}{2}\left(\mathbf{B}\left(\mathbf{v}_{\mathbf{J}}, \mathbf{D}_{n F}^{-1}\right) \mathbf{J}(t), \mathbf{J}(t)\right) .
$$

Since $\mathbf{B}\left(\mathbf{v}_{\mathbf{J}}, \mathbf{D}_{n F}^{-1}\right)$ is negative semidefinite

$$
\frac{\tau}{2} \frac{d}{d t}\left(\mathbf{D}_{n F}^{-1} \mathbf{J}(t), \mathbf{J}(t)\right)+\alpha_{n F,-1}\left(\|\mathbf{J}(t)\|_{0}^{2}+\frac{\tau}{2}|\mathbf{J}(t)|_{\mathbf{v} \mathbf{J}, \partial \Omega}^{2}\right) \leqslant-(\nabla c(t), \mathbf{J}(t)) .
$$

Owing to the Poincaré inequality, we can choose a positive $\epsilon$ such that

$$
\frac{1}{2 \epsilon}\|c(t)\|_{0}^{2} \leqslant \frac{\alpha_{F}}{2}\|\nabla c(t)\|_{0}^{2} .
$$

Inserting (39) in (32), integrating (32) and (38) over $[0, t]$ and summing the resulting inequalities term by term, we get

$$
\begin{aligned}
& \frac{\alpha_{n F,-1} \tau}{2}\|\mathbf{J}(t)\|_{0}^{2}+\frac{1}{2}\|c(t)\|_{0}^{2}+\frac{\alpha_{F}}{2} \int_{0}^{t}\|\nabla c(s)\|_{0}^{2} d s \\
& \quad+\alpha_{n F,-1}\left(\int_{0}^{t}\|\mathbf{J}(s)\|_{0}^{2} d s+\frac{\tau}{2} \int_{0}^{t}|\mathbf{J}(s)|_{\mathbf{v}_{\mathbf{J}}, \partial \Omega}^{2} d s\right) \leqslant+\frac{1}{2}\left(\left\|c_{0}\right\|_{0}^{2}+\tau \alpha_{n F}\left\|\mathbf{J}_{0}\right\|_{0}^{2}\right)+\frac{\epsilon}{2} \int_{0}^{t}\|f(s)\|_{0}^{2} d s
\end{aligned}
$$

that leads to $(31)$.

Remark 1. The technical assumption (27) is a requirement in the previous proof. However, this assumption is valid in a considerable range of cases. Indeed, if we recall [8], the flux velocity is the velocity associated with the flow of a fluid. Bearing this is mind, in this particular (although wide) setting, it is subjected to possibly homogeneous Dirichlet or zero normal trace boundary conditions, allowing the flux velocity to satisfy condition (27). On another scenario, assuming for instance that the velocity is given by Darcy's law, full Neumann homogeneous boundary conditions (for the associated pressure) also translate in the satisfaction of this condition. 
Remark 2. The negative semidefiniteness of $\mathbf{B}\left(\mathbf{v}_{\mathbf{J}}, \mathbf{D}_{n F}^{-1}\right)$ is a condition that steams from the presence of a nonzero flux velocity and its physical meaning is not clear in the more general setting. However, in the case that $\mathbf{D}_{n F}$ is constant, this condition is satisfied if we assume that $\nabla \cdot \mathbf{v}_{\mathbf{J}}=0$. As already pointed out in Remark 1, given that the flux velocity can be associated with a fluid flow, this condition holds for incompressible fluids.

An estimate similar to (31) could be obtained considering different norm/semi-norm for the flux, namely $\|\mathbf{J}(t)\|_{0, \mathbf{D}_{n F}^{-1}}:=\left(\mathbf{D}_{n F}^{-1} \mathbf{J}(t), \mathbf{J}(t)\right)$ and $|\mathbf{J}(t)|_{\mathbf{v}_{\mathbf{J}}, \partial \Omega}^{2}=\int_{\partial \Omega}|\mathbf{J}(t)|^{2} \mathbf{v}_{\mathbf{J}} \cdot \mathbf{n} d s$, for which the new estimate would read

$$
\begin{aligned}
&\|c(t)\|_{0}^{2}+\tau\|\mathbf{J}(t)\|_{0, \mathbf{D}_{n F}^{-1}}^{2}+\alpha_{F} \int_{0}^{t}\|\nabla c(s)\|_{0}^{2} d s \\
&+2 \int_{0}^{t}\|\mathbf{J}(s)\|_{0, \mathbf{D}_{n F}^{-1}}^{2} d s+\tau \int_{0}^{t}|\mathbf{J}(s)|_{\mathbf{v}_{\mathbf{J}}, \partial \Omega, \mathbf{D}_{n F}^{-1}}^{2} d s \leqslant\left\|c_{0}\right\|_{0}^{2}+\tau\left\|\mathbf{J}_{0}\right\|_{0}^{2}+\epsilon \int_{0}^{t}\|f(s)\|_{0}^{2} d s .
\end{aligned}
$$

\section{Numerical simulation}

In this section we apply a finite element method to approximate the solution of (29) in a twodimensional setting. This will serve to illustrate the behaviour of the solution of the coupled model, under different choices of parameters.

We start by introducing the numerical method. Let $N$ be a positive integer and $\Delta t$ a positive real such that $N=\frac{T}{\Delta t}$. The points $t_{i}=i \Delta t, i=0, \ldots, N$ define a partition in $[0, T]$. Let $h$ denote a positive real and $\mathcal{T}_{h}$ a quasi-uniform mesh on $\Omega$, with mesh size $h$ and $V_{h}$ the space of piecewise linear polynomials built on $\mathcal{T}_{h}$. Let $V_{h, 0}=V_{h} \cap H_{\Gamma}^{1}(\Omega)$ and $P_{h}: L^{2}(\Omega) \longrightarrow V_{h}$ denote the $L^{2}$ projection operator onto $V_{h}$. The corresponding vectorial versions of $V_{h}$ and $P_{h}$ are represented by bold letters.

\subsection{Numerical method}

The finite element approximation of (29) reads as: given $c_{h}^{0}=P_{h} c_{0}$ and $\mathbf{J}_{h}^{0}=\mathbf{P}_{h} \mathbf{J}_{0}$, find $c_{h}^{n+1} \in V_{h, 0}$ and $\mathbf{J}_{h}^{n+1} \in \mathbf{V}_{h}$ such that

$$
\begin{array}{rlrl}
\frac{1}{\Delta t}\left(c_{h}^{n+1}, v_{h}\right)+\left(\mathbf{D}_{F} \nabla c_{h}^{n+1}-\mathbf{J}_{h}^{n+1}, \nabla v_{h}\right) & =\left(P_{h} f\left(t_{n+1}\right), v_{h}\right)+\frac{1}{\Delta t}\left(c_{h}^{n}, v_{h}\right), & & \forall v_{h} \in V_{h, 0} \\
\left(\frac{\tau}{\Delta t}+1\right)\left(\mathbf{J}_{h}^{n+1}, \mathbf{w}_{h}\right)+\tau\left(\left(\mathbf{v}_{\mathbf{J}} \cdot \nabla\right) \mathbf{J}_{h}^{n+1}, \mathbf{w}_{h}\right) & & \\
+\left(\mathbf{D}_{n F} \nabla c_{h}^{n+1}, \mathbf{w}_{h}\right) & =\frac{\tau}{\Delta t}\left(\mathbf{J}_{h}^{n}, \mathbf{w}_{h}\right), & & \forall \mathbf{w}_{h} \in \mathbf{V}_{h} .
\end{array}
$$

Remark 3. Method (42) treats coupled problem (29) fully implicitly and we expect that this scheme benefits from reasonable stability properties. It can be shown that (42) satisfies a discrete version of (31).

Remark 4. The equation for the nonfickian flux is, assuming the term $-\mathbf{D}_{n F} \nabla c$ acts as a source, of convection-reaction type. Standard finite element formulations as (42) might not perform very well for certain choices of $\tau$ and $\mathbf{v}_{\mathbf{J}}$. In order to increase the robustness of the 
numerical method, we add an interior penalty stabilization term, see [4], to the left hand side of the second equation of (42)

$$
j\left(\mathbf{J}_{h}, \mathbf{w}_{h} ; \mathbf{v} \mathbf{J}\right):=\gamma \sum_{F \in \mathcal{F}_{I}} \int_{F} h_{F}^{2}|\mathbf{v} \mathbf{J} \cdot \mathbf{n}| \llbracket \nabla \mathbf{J}_{h} \rrbracket_{F} \cdot \llbracket \nabla_{\mathbf{w}_{h} \rrbracket_{F}} d s, \forall \mathbf{J}_{h}, \mathbf{w}_{h} \in \mathbf{V}_{h},
$$

where $\mathcal{F}_{I}$ denotes the set of interior faces of the triangulation $\mathcal{T}_{h}, \llbracket \cdot \rrbracket_{F}$ denotes the usual jump function across the face $F, h_{F}^{2}$ is the length (for $d=2$, or area for $d=3$ ) of face $F$ and $\gamma>0$ is a parameter.

\subsection{Comparison of different flux behaviours}

In this section we try to illustrate the behaviour of some of the solutions of system (13)-(12).

For our first numerical simulation, let us start by fixing some data and initial conditions. Let $\Omega=(0,1)^{2}$. We consider $\mathbf{D}_{F}=\mathbf{D}_{n F}=\mu_{n F} \mathbf{I}=0.01 \mathbf{I}$, where $\mathbf{I}$ denotes the identity matrix, and the following initial conditions

$$
c_{0}(x, y)=e^{-\frac{(x-0.5)^{2}+(y-0.5)^{2}}{0.02}}, \quad \mathbf{J}_{0}(x, y)=-\mu_{n F} \nabla c_{0}(x), \quad \forall x \in \Omega .
$$

The initial concentration profile is plotted in Figure 1. We consider homogeneous Dirichlet
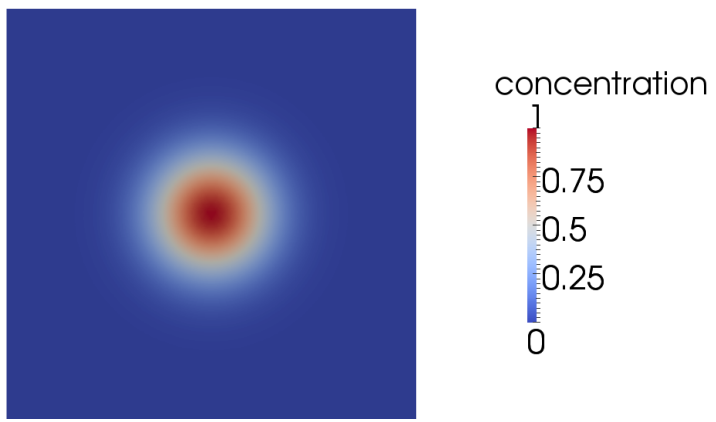

Figure 1: Concentration profile at $t=0$.

boundary conditions at $x=0$ and $x=1$ and homogeneous Neumann conditions at $y=0$ and $y=1$. The discretization parameters are chosen as $h=0.02$ and $\Delta t=0.005$. We start by simulating the case $\mathbf{v}_{\mathbf{J}}=\mathbf{0}$ and consider different values for $\tau$ : $\tau=0,10^{-3}, \tau=10^{-2}$ and $\tau=10^{-1}$. Note that the first test, $\tau=0$ corresponds to the pure fickian regime. From Figure 2 , the increase of $\tau$ does not seem to have a substantial effect on the solution. It is however noticeable that for values of $\tau$ big enough, the numerical solution obtained is nonphysical, which does not happen for small values of $\tau$. This can be explained by the fact that, for large enough $\tau$ (we can think of the limiting case $\tau \longrightarrow+\infty$ ), the solution of this problem is the solution of a wave type equation. In fact, from (22), taking $\tau$ big enough, $\mathbf{v}_{\mathbf{J}}=\mathbf{0}$ and $f=0$, we conclude that the solution $c$ of the differential system is the solution of the wave equation. Therefore, the behaviour exhibited in Figure 2(d) is in perfect agreement with the behaviour of the exact solution of the system. In this example, we do not observe any different decay or delay in the concentration when comparing with the fickian case.

We consider now another example. Let us take another initial condition and choice of parameters; let $\Omega$ denote the same domain as before and

$$
c_{0}(x, y)=100 x(1-x)[1+H(y-0.4)(-5(y-0.4))-H(y-0.6)(1-5(y-0.4))],
$$




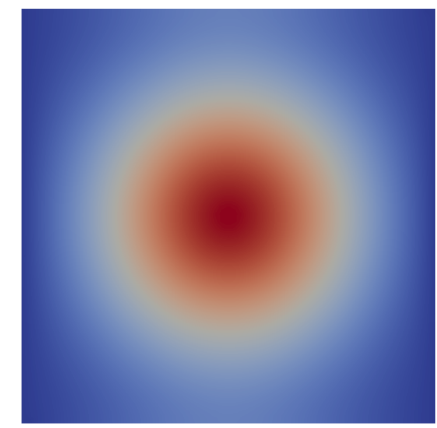

concentration
0.177071
0.16
0.12
0.08
0.04
$6.033 e-5$

(a) Pure fickian diffusion: case $\tau=0$

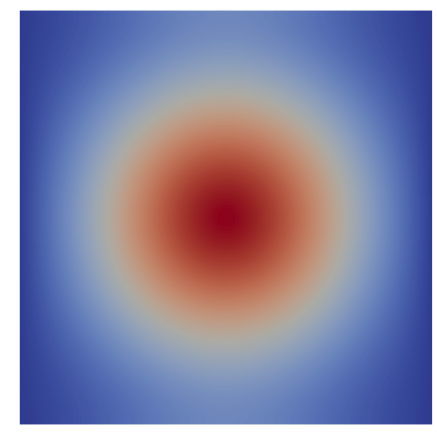

(c) Case $\tau=0.1$ concentration

0.165847

0.16

0.12

0.08

0.04

$5.886 \mathrm{e}-5$

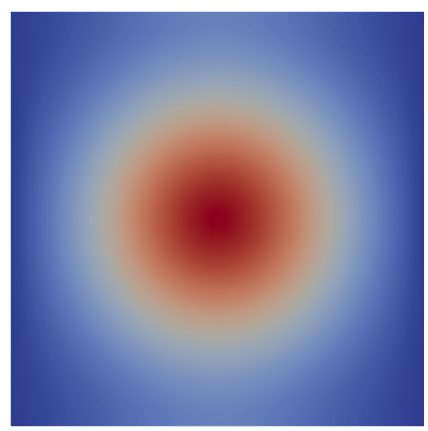

concentration

0.176019

0.16

0.12

0.08

0.04

$6.036 e-5$

(b) Case $\tau=0.01$

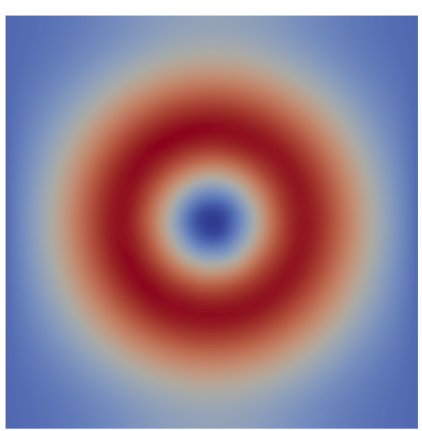

(d) Case $\tau=1$ concentration

0.135336

0.12

0.08

0.04

0

$-0.021663$

Figure 2: Plots of concentration profiles at $t=0.5$ for different choices of $\tau$.

where $H$ denotes the Heaviside function. The same boundary condition setting as in the previous example is chosen. We take the fickian and nonfickian diffusion tensors as $\mathbf{D}_{F}=\mathbf{D}_{n F}=\mathbf{I}$. To illustrate the behaviour of the parameter $\tau$ (while still considering a zero nonfickian flux velocity), we run several simulations ( $h=0.01, \Delta t=10^{-4}$ ) corresponding to $\tau=0$ (pure fickian diffusion), $\tau=10^{-3}$ and $\tau=10^{-2}$.

In Figure 3(a) we plot the concentration profile at $t=0$. For the different values of $\tau$, the evolution of the concentration is very similar, see Figures 3(b)-3(d). We can however notice that the larger $\tau$ is, the faster the diffusion process seems to occur. To further investigate this phenomena, we looked at the maximum and average concentration as a function of time, see Figures 4(a) and 4(b). From Figure 4(a), at first the diffusion is delayed by the increased parameter $\tau$ (although the difference is not very significant), but as time evolves, the diffusion accelerates and the concentration decreases faster than in the fickian case. The latter behaviour is also evident in the average concentration plot, see Figure 4(b).

Finally we consider again the first simulation in this section, but with $\mathbf{v}_{\mathbf{J}}$ given as

$$
\mathbf{v} \mathbf{J}=\alpha(x, 2(y-1))^{T}, \forall(x, y) \in \Omega,
$$

where $\alpha>0$ is a constant parameter used to control the maximum magnitude of $\mathbf{v}_{\mathbf{J}}$. In Figure 5 we plot the numerical solution obtained with $h=0.02, \Delta t=0.02, T=0.08, \tau=10^{-2}, \mu=$ $0.1, \gamma=0.1$. Only for large values of $\alpha$ we can notice a different behaviour induced by the extra convective flux term. As in the previous simulation, the choice of the parameter $\mathbf{v}_{\mathbf{J}}$ has an impact on the profile of the concentration $c$. While in the previous case we noticed an acceleration in the diffusion process, the consideration of a flux velocity induces some sort of convective phenomenon to the concentration field, which, in this case, breaks the symmetry of the solution. 


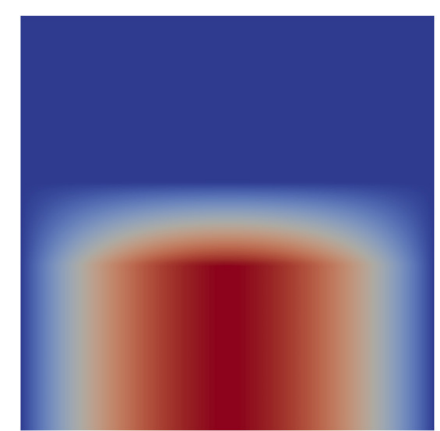

(a) Initial concentration

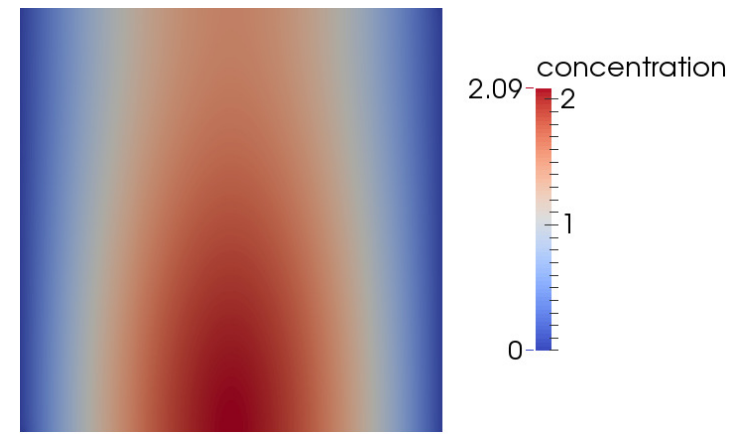

(c) Case $\tau=0.01$

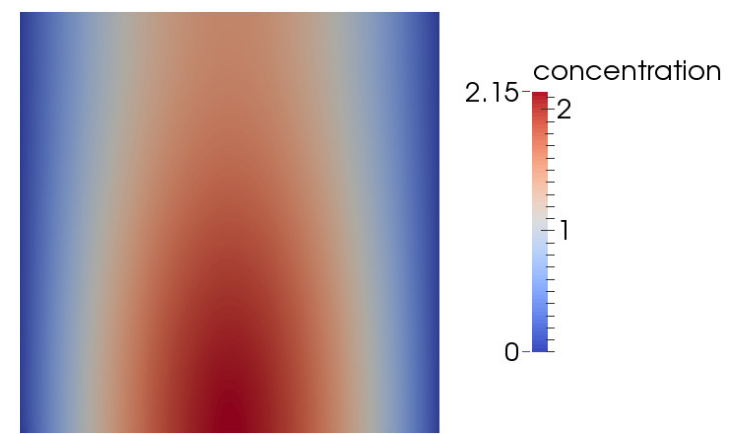

(b) Pure fickian diffusion: case $\tau=0$

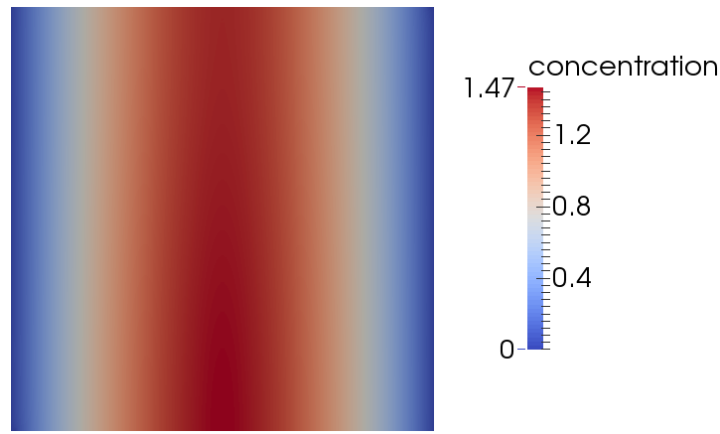

(d) Case $\tau=0.1$

Figure 3: Plots of concentration profiles at $t=0.1$ for different choices of $\tau$.

\section{Conclusions}

We have considered a coupled system of equations to model a diffusion process presenting a fickian and nonfickian mass flux contributions. This model introduces a memory effect in time and space for the flux.

In some special cases, we showed that the coupled problem is the same as others already proposed in the literature. Also, the energy estimates obtained for the coupled problem are consistent with the ones found for those other equivalent formulations. A finite element method was implemented to illustrate the different behaviour of the various effects of memory in time and space.

It is our goal to apply the model studied in this paper to model diffusion (and advection) in porous media. In this case, this system should be coupled with Darcy's law for the velocity and an elliptic equation for the pressure (for incompressible flows).

\section{Acknowledgements}

This work has been partially supported by the Centre for Mathematics of the University of Coimbra and Fundação para a Ciência e a Tecnologia, through European program COMPETE/FEDER and by the project UTAustin/MAT/0066/2008. 


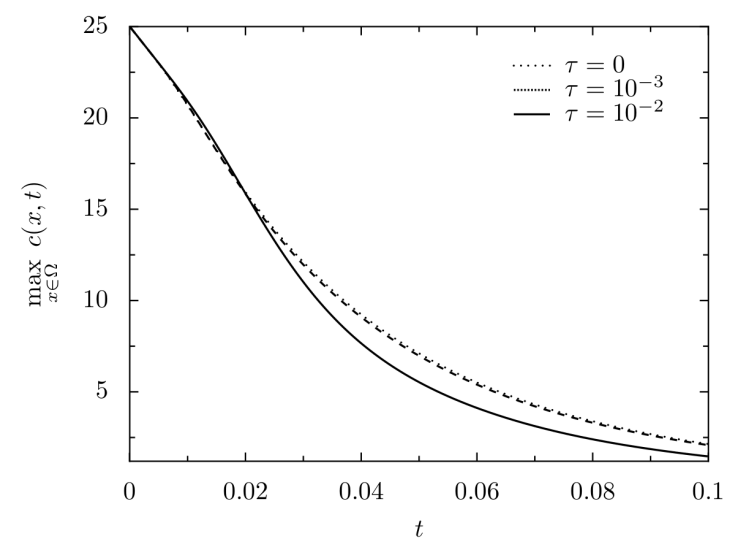

(a) Maximum concentration over time

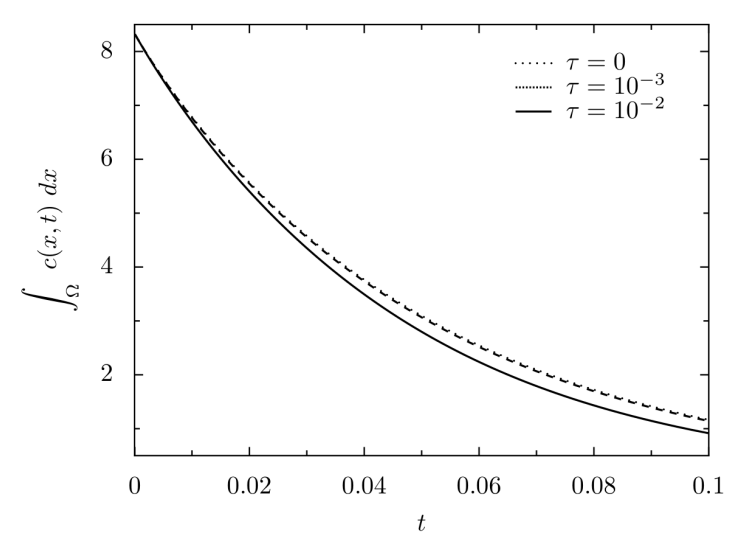

(b) Average concentration over time

Figure 4: Plots of maximum and average concentration along time for different choices of $\tau$.

\section{References}

[1] A. Araújo, J. R. Branco, and J. A. Ferreira. On the stability of a class of splitting methods for integro-differential equations. Appl. Numer. Math., 59(3-4):436-453, March 2009.

[2] D. L. Bernik, D. Zubiri, M. E. Monge, and R.M. Negri. New kinetic model of drug release from swollen gels under non-sink conditions. Colloids Surf. A: Physicochem. Eng. Asp., 273:165-173, 2006.

[3] J. R. Branco, J. A. Ferreira, and P. de Oliveira. Numerical methods for the generalized fisher-kolmogorov-petrovskii-piskunov equation. Appl. Numer. Math., 57(1):89-102, 2007.

[4] Erik Burman, Alfio Quarteroni, and Benjamin Stamm. Interior penalty continuous and discontinuous finite element approximations of hyperbolic equations. Journal of Scientific Computing, 43(3):293-312, 2010.

[5] J. A. Ferreira and P. De Oliveira. Memory effects and random walks in reaction-transport systems. Appl. Anal., 86(1):99-118, 2007.

[6] J. A. Ferreira, P. de Oliveira, and P. M. da Silva. Analitycs and numerics of drug release tracking. Journal of Computational and Applied Mathematics, 236:3572-3583, 2012.

[7] J. A. Ferreira, P. de Oliveira, and P. M. da Silva. Reaction-diffusion in viscoelastic materials. Journal of Computational and Applied Mathematics, 236:3783-3795, 2012.

[8] S. M. Hassahizadeh. On the transient non-fickian dispersion theory. Transp. Porous Media, 23:107-124, 1996.

[9] Y. Lin. Semi-discrete finite element approximations for linear parabolic integro-differential equations with integrable kernels. J. Integral Equations Appl., 10:51-83, 1998.

[10] Y. Lin, V. Thomée, and L. B. Wahlbin. Ritz-volterra projections to finite-element spaces and applications to integro-differential and related equations. SIAM J. Numer. Anal., 28(4):1047-1070, August 1991.

[11] C. Maas. A hyperbolic dispersion equation to model the bounds of a contaminated groundwater body. J. Hydrol., 226(34):234 - 241, 1999. 


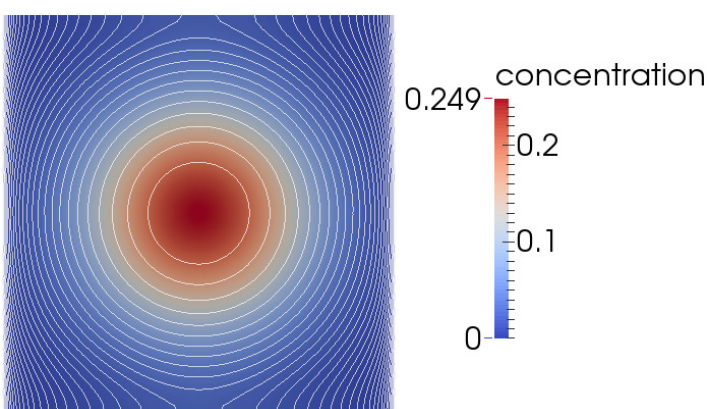

(a) Case $\alpha=0.1$

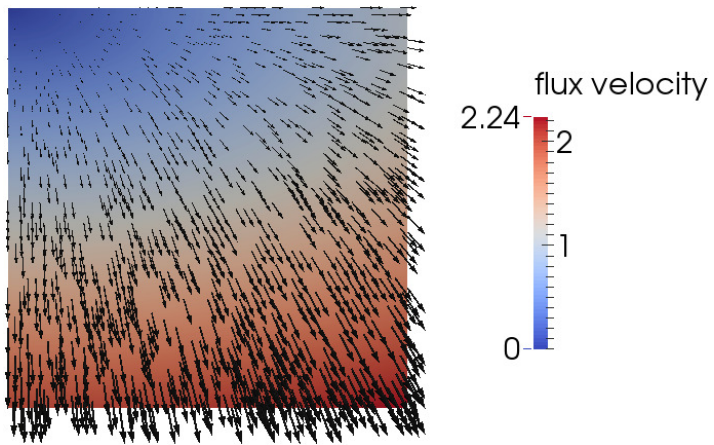

(c) Magnitude of nonfickian flux velocity and vector representation

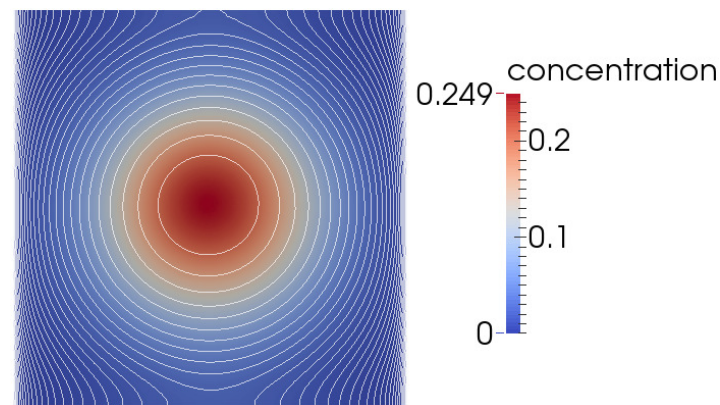

(b) Case $\alpha=1$

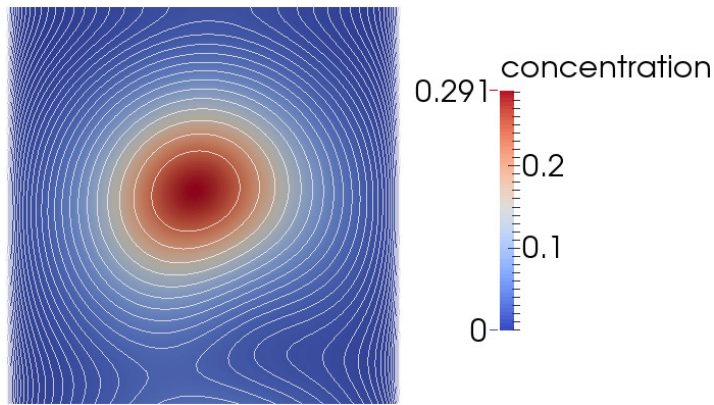

(d) Case $\alpha=10$

Figure 5: Plots of concentration profiles at $t=0.08$ for different choices of $\alpha$. The contours correspond to the same values for all figures.

[12] S. P. Neuman and D. M. Tartakovsky. Perspective on theories of non-fickian transport in heterogeneous media. Adv. Water Resour., 32(5):670 - 680, 2009.

[13] A. K. Pani and T. E. Peterson. Finite element methods with numerical quadrature for parabolic integro-differential equations. SIAM J. Numer. Anal., 33(3):1084-1105, June 1996.

[14] A. E. Scheidegger. Typical solutions of the differential equations of statistical theories of flow through porous media. Trans. Amer. Geophys. Union, 39:929-932, 1958.

[15] R. K. Sinha, R. E. Ewing, and R. D. Lazarov. Some new error estimates of a semidiscrete finite volume element method for a parabolic integro-differential equation with nonsmooth initial data. SIAM J. Numer. Anal., 43(6):2320-2343, January 2006.

[16] O. D. L. Strack. A mathematical model for dispersion with a moving front in groundwater. Water Resour. Res., 28:2973-2980, 1992.

[17] V. Thomée and N-Y. Zhang. Error estimates for semidiscrete finite element methods for parabolic integro-differential equations. Math. Comp., 53:121-139, 1989.

[18] A. F. B. Tompson. A new functional form for the dispersive flux in porous media. Water Resour. Res., 24:1939-1947, 1988. 